\title{
Direct observation of the influence of the As-Fe-As angle on the $T_{c}$ of superconducting $\mathrm{SmFeAsO}_{1-x} \mathrm{~F}_{x}$
}

\author{
G. Garbarino,,${ }^{1,}$ R. Weht, ${ }^{2,3}$ A. Sow ${ }^{4}$ A. Sulpice,${ }^{4}$ P. Toulemonde, ${ }^{4}$ M. Álvarez-Murga,${ }^{1,4}$ P. Strobel, ${ }^{4}$ P. Bouvier, ${ }^{5,1}$ \\ M. Mezouar, ${ }^{1}$ and M. Núñez-Regueiro ${ }^{4}$ \\ ${ }^{1}$ European Synchrotron Radiation Facility (ESRF), 6 rue Jules Horowitz, BP 220, F-38043 Grenoble Cedex, France \\ ${ }^{2}$ Gerencia de Investigación y Aplicaciones, Comisión Nacional de Energía Atómica (CNEA), Avda. General Paz y Constituyentes, \\ 1650-San Martín, Argentina \\ ${ }^{3}$ Instituto Sabato, Universidad Nacional de San Martín-CNEA, 1650 - San Martín, Argentina \\ ${ }^{4}$ Institut NEEL, CNRS and Université Joseph Fourier, BP 166, 25 avenue des Martyrs, F-38042 Grenoble Cedex 9, France \\ ${ }^{5}$ Laboratoire des Matériaux et du Génie Physique, CNRS UMR 5628, Grenoble Institute of Technology, MINATEC, 3 parvis Louis Néel, \\ 38016 Grenoble, France
}

(Received 10 January 2011; revised manuscript received 15 February 2011; published 11 July 2011)

\begin{abstract}
The electrical resistivity, crystalline structure, and electronic properties calculated from the experimentally measured atomic positions of the compound $\mathrm{SmFeAsO}_{0.81} \mathrm{~F}_{0.19}$ have been studied up to pressures $\sim 20 \mathrm{GPa}$. The correlation between the pressure dependence of the superconducting transition temperature $\left(T_{c}\right)$ and crystallographic parameters on the same sample shows clearly that a regular $\mathrm{FeAs}_{4}$ tetrahedron maximizes $T_{c}$ through optimization of carrier transfer to the FeAs planes as indicated by the evolution of the electronic band structures.
\end{abstract}

DOI: 10.1103/PhysRevB.84.024510

PACS number(s): 74.70.Xa, 61.50.Ks, 62.50.-p, 72.80.Ga

\section{INTRODUCTION}

The discovery of superconductivity ${ }^{1-4}$ in layered compounds of iron in tetrahedral coordination (TIC) with superconducting critical temperatures $\left(T_{c}\right)$ reaching up to $56 \mathrm{~K}$ has refreshed the interest in high-temperature superconductors (HTSCs). In particular, in the search for a common pattern for HTSCs, the similarities and differences between cuprates and TICs are now being intensively investigated. One example is the influence of the angle between the cations and anions in the active planes, $\mathrm{CuO}_{2}$ and $\mathrm{FeAs}$, which can have a profound impact on their electronic properties. ${ }^{5-8}$ For cuprates, the rule of thumb says that flatter $\mathrm{Cu}-\mathrm{O}-\mathrm{Cu}$ buckling angles $\left(\sim 180^{\circ}\right)$ give higher $T_{c}$ 's, e.g., flat-plane record bearing $\mathrm{Hg}$ cuprates, ${ }^{9} 214$ materials, ${ }^{10}$ with notable exceptions as 123 compounds due to their unique structure. ${ }^{11}$ For TICs it has been reported ${ }^{12-15}$ that regular tetrahedral AsFeAs angles $\left(109.47^{\circ}\right)$ yield the highest $T_{c}$ 's. Different theoretical models ${ }^{7,8}$ have been proposed to explain this dependence, all based on the great sensitivity of electronic properties with atomic positions. In this work, we report a simultaneous analysis of superconducting properties, crystal structure, and electronic energy-band structures on the same $\mathrm{SmFeAsO}_{1-x} \mathrm{~F}_{x}(x \approx$ $0.19)$ compound as a function of pressure. The fact that we use pressure as a control parameter on the same sample is of extreme importance considering that it is a clean mechanism to modify the structure; it avoids spurious impurities or other effects associated with the atomic replacement. Our results verify the relation between the $T_{c}$ and the optimum AsFeAs angle; they also provide an explanation that notably differs from those previously proposed.

\section{EXPERIMENTAL TECHNIQUES}

$\mathrm{SmFeAsO}_{1-x} \mathrm{~F}_{x}$ samples were prepared by a high-pressure, high-temperature treatment using a "belt"-type high-pressure apparatus. $\mathrm{Sm}, \mathrm{Fe}, \mathrm{Fe}_{2} \mathrm{O}_{3}, \mathrm{As}$, and $\mathrm{SmF}_{3}$, powders were mixed together as precursors and pressed into the form of cylindrical pellets. For the synthesis, the pellet was introduced in a homemade boron nitride crucible which was surrounded by a cylindrical graphite resistive heater, and the whole assembly was placed in the pyrophyllite gasket. The samples were treated at $6 \mathrm{GPa}, 1000^{\circ} \mathrm{C}-1100^{\circ} \mathrm{C}$ for $4 \mathrm{~h}$, then quenched to room temperature. The $\mathrm{x}$-ray diffraction pattern at room pressure and temperature showed a very good sample quality with $<0.5 \%$ impurity phases. Superconductivity was checked by resistivity and ac susceptibility measurement showing an onset of superconductivity at $54 \mathrm{~K}$. The fluorine composition was obtained through the comparison of the cell volume at ambient conditions of our sample, $V_{0}=130.89(5) \AA^{3}$, with the literature data of the parent compound ${ }^{16}$ and different doping values. ${ }^{17}$ Assuming a linear dependence of $V_{0}$ with fluorine doping up to $\sim 20 \%$, the effective composition was estimated to be $x \approx 0.19$.

The $\mathrm{x}$-ray diffraction studies were performed on the $\mathrm{SmFeAsO}_{0.81} \mathrm{~F}_{0.19}$ powder samples at the ID27 high-pressure beam line of the European Synchrotron Radiation Facility using a monochromatic beam $\left(\lambda=0.3738 \AA^{3}\right)$ focused to $3 \times 2 \mu \mathrm{m}^{2}$. The diamond anvil cells with $600 \mu \mathrm{m}$ cullet diamonds with stainless steel gasket and two different pressure media were used. In the low-pressure range $(P<3 \mathrm{GPa})$ a $4: 1$ methanol-ethanol mixture assured a detailed analysis of the pressure dependence of the structural parameters, while using neon guaranteed the hydrostatic conditions up to the highest applied pressure, $P<20 \mathrm{GPa}$. Pressure was measured through the shift of the fluorescence line of the ruby. All the structural studies were done at ambient temperature. The diffraction patterns were collected with a CCD camera and the intensity vs $2 \theta$ patterns were obtained using the FIT2D software. ${ }^{18} \mathrm{~A}$ complete Rietveld refinement was done with the GSAS-EXPGUI package. ${ }^{19}$

The electrical resistance measurements were performed using a Keithley 2400 source meter and a Keithley 2182 
nanovoltmeter. Pressure measurements, 0.2-7 GPa (between 4.2 and $300 \mathrm{~K}$ ), were done in a tungsten carbide Bridgman anvil apparatus using a pyrophillite gasket and two steatite disks as the pressure medium. ${ }^{20}$

\section{RESULTS AND DISCUSSION}

Figure 1(a) shows the 3-GPa room temperature diffraction pattern together with its Rietveld refinement, obtained using a

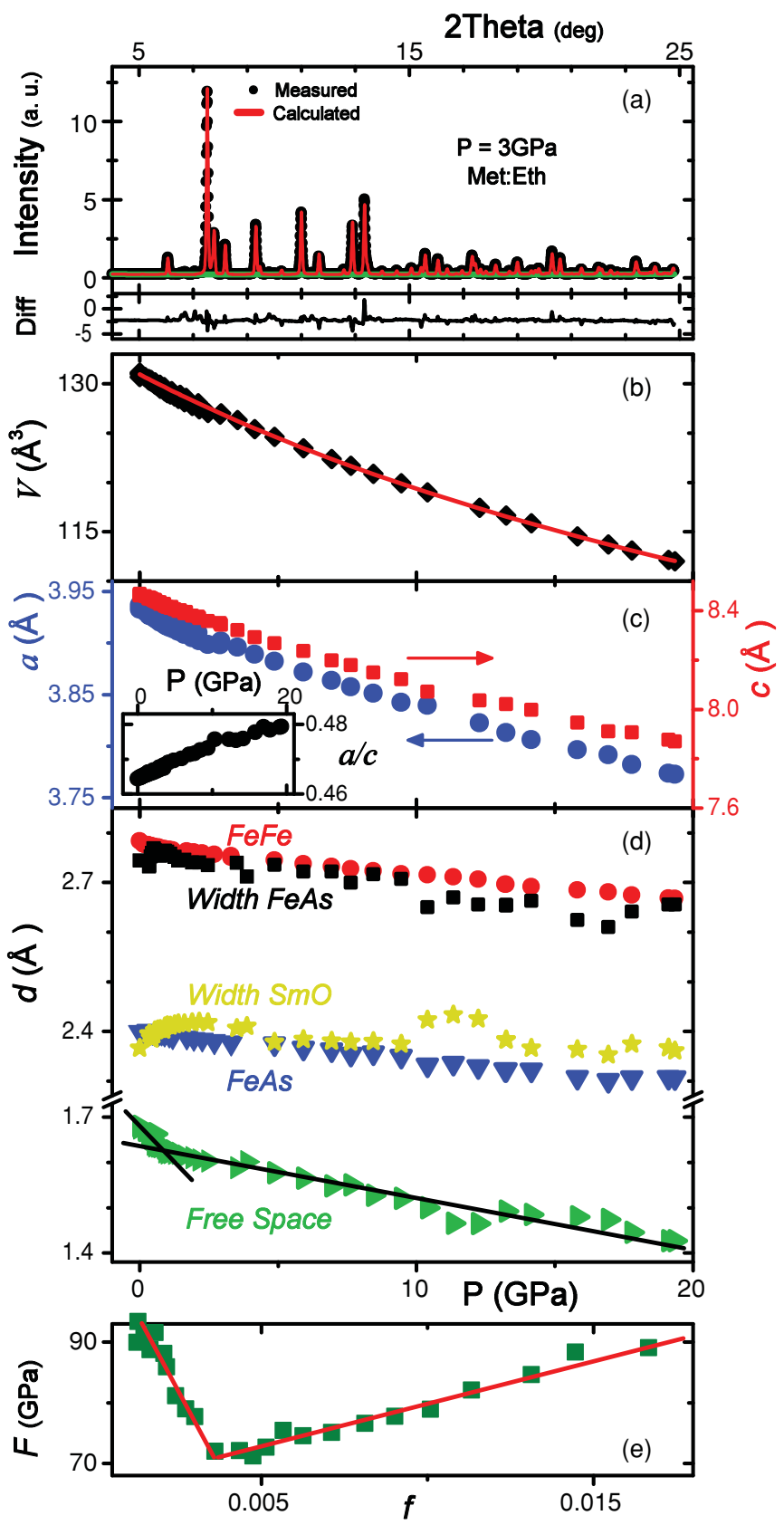

FIG. 1. (Color online) (a) X-ray diffraction pattern measured at $3 \mathrm{GPa}$ of the $\mathrm{SmFeAsO}_{0.81} \mathrm{~F}_{0.19}$ (black filled circles). The red (gray) line represents the Rietveld refinement using a tetragonal $P 4 / \mathrm{nmm}$ structure, while the black line is the difference between the model and the data. Pressure dependence of the (b) volume, (c) lattice parameters, and (d) interatomic distances of the $\mathrm{SmFeAsO}_{0.81} \mathrm{~F}_{0.19}$ sample. The inset of panel (c) shows the monotonic evolution of the $a / c$ ratio. (e) Normalized pressure, $F$, vs Eulerian strain $f$. The lines show linear fits of the regions of the compression curves. tetragonal $P 4 / \mathrm{nmm}$ unit cell and refining scaling factor, lattice parameters, profile shapes, and the atomic positions of the As and $\mathrm{Sm}$, while the $\mathrm{O} / \mathrm{F}$ site was considered fully occupied. The pressure evolution of the diffraction patterns up to $20 \mathrm{GPa}$ does not show any evidence of phase transition. This is a common behavior in other structure studies of the same 1111 family under pressure at room temperature. ${ }^{21}$

The pressure dependence of the volume, $V$, lattice parameters, $a$ and $c$ of the unit cell and the $a / c$ ratio are given in Figs. 1(b) and 1(c), respectively. A third-order BirchMurnaghan equation of state was used to determine the bulk modulus $K_{0}$ and its pressure dependence $K_{0}^{\prime}=\partial K_{0} / \partial p .^{22}$ The unit cell volume at ambient pressure, $V_{0}$, was fixed at the measured value $V_{0}=130.89(5) \AA^{3}$, while $K_{0}=88.9(8) \mathrm{GPa}$ and $K_{0}^{\prime}=4.2(1)$ were obtained by the fit. Similar $K_{0}$ and $K_{0}^{\prime}$ values have been reported in other compounds of the same 1111 family. ${ }^{21,23}$ In the inset of Fig. 1(c) the pressure evolution of the $a / c$ ratio is presented, and clearly, a monotonous function (particularly a linear one) can be used to describe its pressure dependence in the entire studied range.

The pressure was gradually increased in the low- $P$ range $(P<3 \mathrm{GPa})$ in order to have a very detailed analysis of the compression of the structure and correlate it with the superconducting and transport properties.

To highlight any anomaly in the volume compression, the data are plotted in Fig. 1(e) in terms of the Eulerian strain, ${ }^{24} f=\left[\left(V_{0} / V\right)^{2 / 3}-1\right] / 2$, and normalized pressure, $F=p /\left[3 f(1+2 f)^{2.5}\right]$, calculated by fixing $V_{0}=130.89(5)$ $\AA^{3}$. A clear kink is indeed observed at $f=0.0037(p \cong$ $0.6 \mathrm{GPa}$ ), that can be correlated with a change in the structure towards a regular $\mathrm{FeAs}_{4}$ tetrahedron and maximal $T_{c}$, as will be discussed later.

The evolution of the structural parameters is presented on Fig. 1(d), showing that the thickness of the FeAs layer, the distances between in-plane Fe ions and between $\mathrm{Fe}$ and As ions (labeled "Width FeAs," "FeFe," and "FeAs," respectively) have a very similar evolution under pressure with a variation of $\sim 4 \%$ at $20 \mathrm{GPa}$. The thickness of the SmO layer slightly changes all over the pressure range; whereas the distance between the FeAs and SmO layers (labeled "Free Space") shows a reduction of $15 \%$ at $20 \mathrm{GPa}$. Similar structural trends with pressure have been reported in other oxypnictide compounds and are characteristic of a layered structure. ${ }^{23}$ At this point it is important to mention the two different linear dependences in the "Free Space" behavior below and above $P \approx 0.6 \mathrm{GPa}$, which are correlated with the kink at $f=0.0037$ in the $F-f$ representation.

Figure 2 shows the temperature dependence of the resistance for different applied pressures up to $7 \mathrm{GPa}$. Under compression the absolute value of the resistance decreases. $T_{c}$, defined as the onset of the superconducting transition, is shown in the inset of Fig. 2 as a function of pressure. It presents a nonmonotonous pressure behavior with a maximum at $0.6 \mathrm{GPa}$, followed by a linear decrease for higher pressure.

The present combination of the structural parameters obtained from $\mathrm{x}$-ray diffraction studies and the transport properties measurements gives very strong information about the important parameter that controls the electronic properties, and particularly in this case the $T_{c}$. Up until now, several reports have pointed out the relevance of the AsFeAs angle and the As height with respect to the Fe layer. For example, 


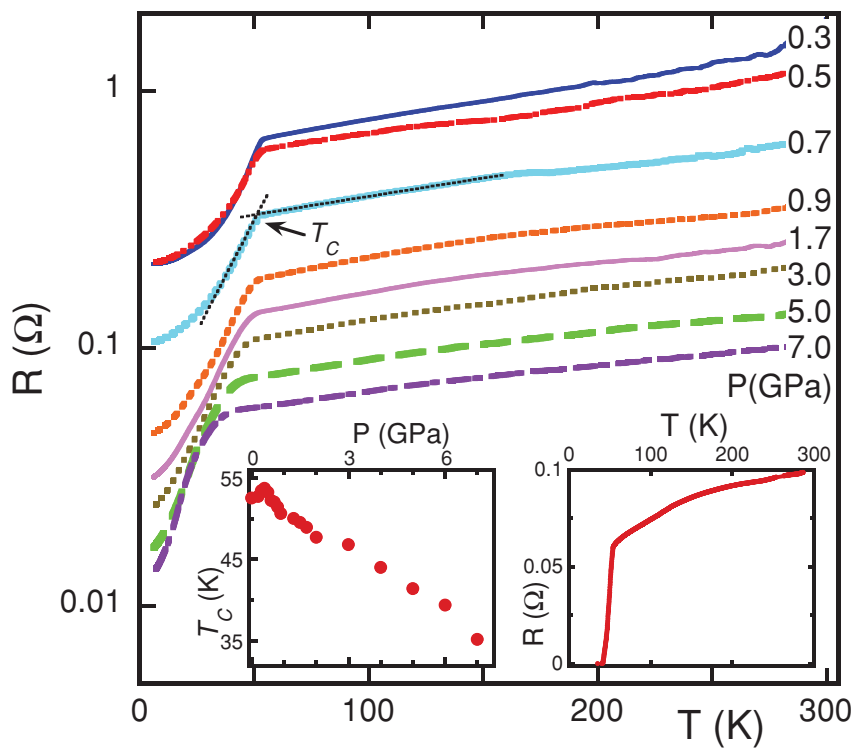

FIG. 2. (Color online) Temperature dependence of the resistance for different applied pressure of the sample $\mathrm{SmFeAsO}_{0.81} \mathrm{~F}_{0.19}$. Left inset: Pressure evolution of $T_{c}$, defined as the onset of the superconducting transition (see intersection of the dotted lines). Right inset: Resistance of the sample at ambient pressure.

Zhao et al. ${ }^{15}$ follow both variations and their correlation as the CeFeAsO system is doped. Also Kimber et al. ${ }^{14}$ describe the variation of the angle with pressure, and compare with results on the pressure variation of $T_{c}$ published elsewhere on a different batch of samples. In both cases, the fact that the measurements are not done on the same samples diminishes the reliability of these reports. We follow the variation of $T_{c}$ and of the structure in the same sample, leaving no margin for uncertainties due to sample preparation, impurities, etc. It is in this respect that our work is more trustworthy. This is done in Fig. 3, which shows the pressure dependence of $T_{c}$ [Fig. 3(a)], the AsFeAs angle [Fig. 3(b)], and the As height [Fig. 3(c)], defined by the distance between As and Fe planes, i.e., $h \mathrm{As}=$ $[z(\mathrm{As})-z(\mathrm{Fe})] c=[z(\mathrm{As})-0.5] c$. The data unambiguously prove that $T_{c}$ reaches its maximum of $55.2 \mathrm{~K}$ at the same pressure as the As height increases up to the optimal value of $1.39 \AA$, and the AsFeAs angle moves to its regular tetrahedral value of $109.47^{\circ}$ [dashed line in Fig. 3(b)]. This is a clear experimental evidence of the correlation between the structural parameters and the superconducting transition on the same sample by means of a clean technique such as pressure.

A special discussion merits study of the relation between the As height ( $h \mathrm{As}$ ) and the AsFeAs angle $(\alpha)$. A simple analysis of the tetragonal structure allows us to deduce the relation between those two structural parameters: $\alpha=$ $2 \tan ^{-1}[(a / 2) / h \mathrm{As}]=2 \tan ^{-1}[(a / c) / 2 z \mathrm{As}-1]$. Considering that the function $g(x)=\tan ^{-1}(x)$ and the pressure evolution of the $a / c$ ratio [inset of Fig. 1(b)] are monotonous, it can be inferred that a nonmonotonous behavior in $\alpha$ is related with the opposite kind of behavior in $z$ As or $h$ As; i.e., $\alpha \propto 1 / z$ As. In addition, in the case of a nondistorted $\mathrm{FeAs}_{4}$ tetrahedron, the relation is reduced to $h \mathrm{As}=a / \sqrt{8}$. As the $a$ lattice parameter is $a \cong 3.95 \AA$ the optimum value for $h \mathrm{As}$ is $1.39 \AA$ and $\alpha=$ $109.47^{\circ}$. This close relation between these two parameters

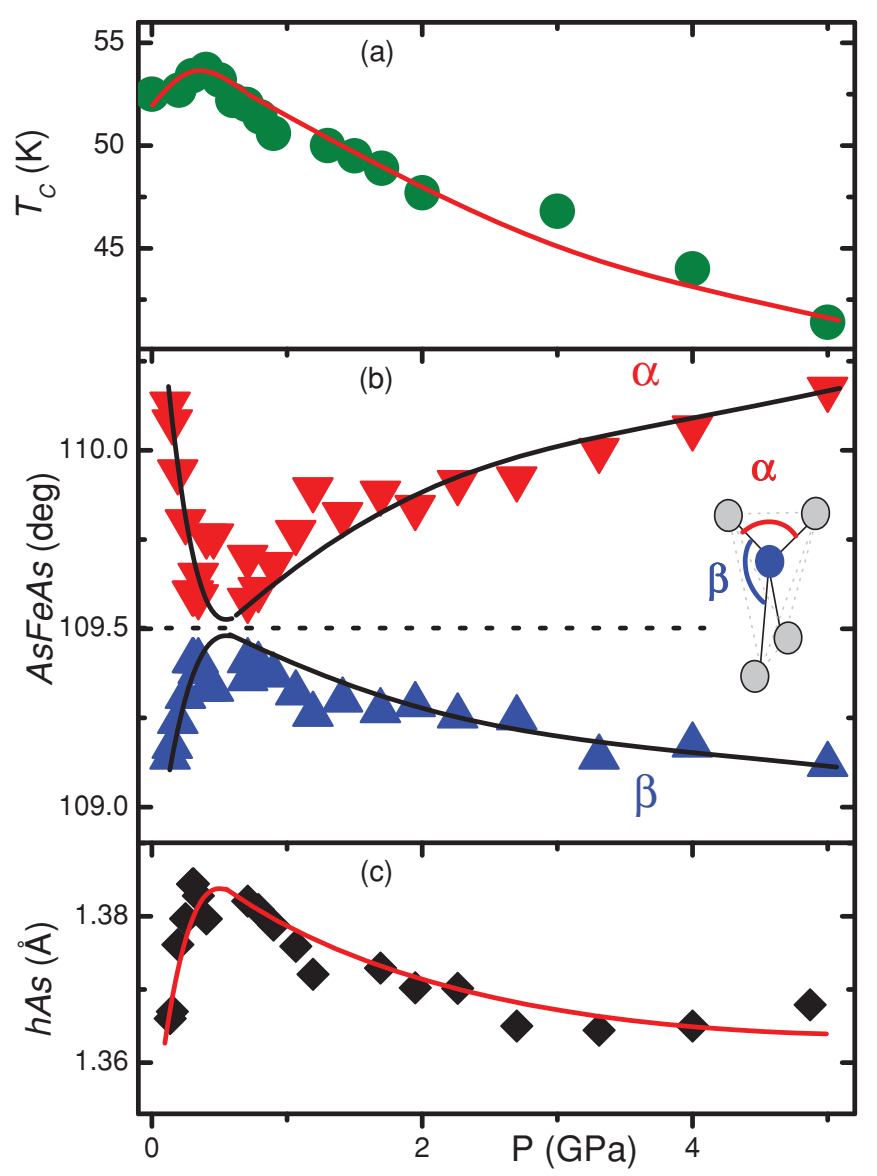

FIG. 3. (Color online) (a) Correlated evolution of $T_{c}$; (b) the tetrahedral AsFeAs angle; (c) the height of the As ( $h$ As) atom relative to the Fe layer of $\mathrm{SmFeAsO}_{0.81} \mathrm{~F}_{0.19}$. The maximum of $T_{c}$ clearly corresponds with the maximum As height and the regular tetrahedral angle $\left[\alpha=\beta=109.47^{\circ}\right.$, represented by the dashed line in (b)].

implies that the structural effects on $T_{c}$ can be explained either by the angle $\alpha$ or the As height $h$ As.

The relation between a regular $\mathrm{FeAs}_{4}$ tetrahedron and maximum $T_{c}$ has been discussed in various numerical studies. ${ }^{7,8}$ A two-orbital-band approach yields a flat band, sensitive to changes in the tetrahedron angle, with a strong peak on the density of states at the Fermi level. ${ }^{8}$ Such a Fermi surface feature can affect nesting, or, in a strong coupling approach, superexchange. Including a third orbital allows symmetry breaking through tetrahedron deformation. Considering the strong hybridization of the five $3 d$ orbitals, it has been pointed out $^{7}$ that a five-orbital model is necessary to analyze the effect of the $h$ As on the spin fluctuations mediated superconductivity. In this framework, the structural parameters modify the nesting scenario between the different bands and consequently the gap function and the $T_{c}$. However, all these considerations may not be applicable here, as they rely on stronger angle variations than those actually measured in our $\mathrm{SmFeAsO}_{1-x} \mathrm{~F}_{x}$.

In order to obtain a detailed microscopic interpretation of the subtle structural effects on the electronic properties and the superconducting transition, $a b$ initio calculations using the actual, measured pressure dependence of the crystal structure and the atomic positions were performed. 
(a) $\mathrm{P}=0.1 \mathrm{GPa}$

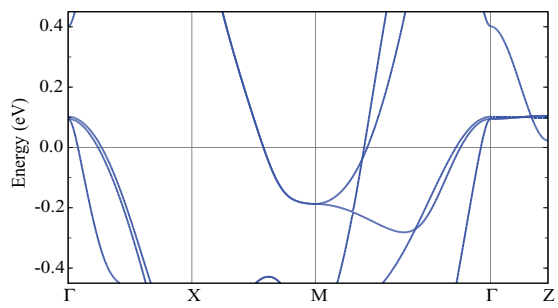

(b) $\mathrm{P}=0.5 \mathrm{GPa}$

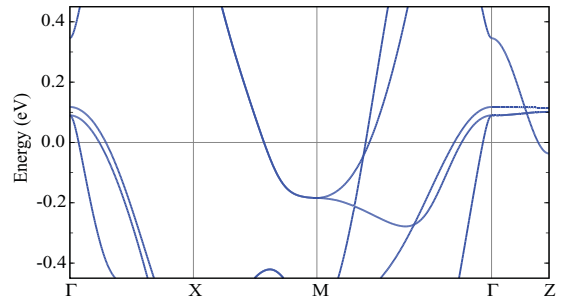

(c) $\mathrm{P}=3.6 \mathrm{GPa}$

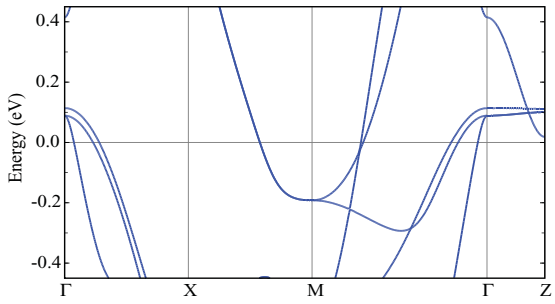

(d)

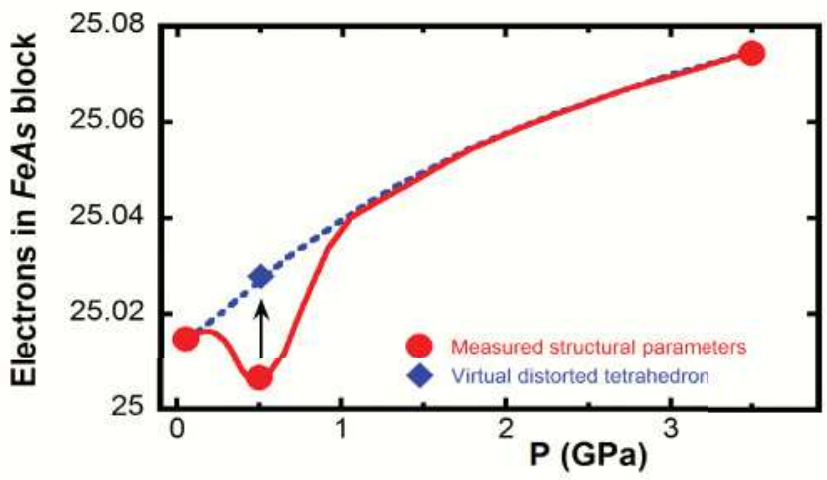

FIG. 4. (Color online) (a), (b), and (c) Pressure evolution of the band structure corresponding to 0.1, 0.5, and 3.6 GPa, respectively. (d) Evolution with pressure of the charge in the FeAs block (red filled circles), calculated using the measured structural parameters and a fictitious distorted tetrahedron (blue diamond). To evaluate this magnitude we use the corresponding LAPW projections inside the muffin-tin spheres. Lines are a guide for the eyes.

All the electronic structure calculations have been carried out using the full-potential linearized-augmented plane wave (FP-LAPW) method as implemented in the WIEN2K code ${ }^{25}$ based on the density functional theory. We considered the Perdew-Burke-Ernzerhof $(\mathrm{PBE})^{26}$ generalized gradient approximations to treat the exchange correlation potential. Sm $4 f$ states were treated as pseudocore states, restricting them to the muffin-tin spheres (for this reason we have considered a quite large muffin-tin radius for $\mathrm{Sm}$, of 2.5 a.u.). Doping was simulated assuming a virtual oxygen atom with a charge of $8+x(x=0.19$ in our case $)$.

Band structures at three representative pressures $(0.1,0.5$, and $3.6 \mathrm{GPa}$ ) are presented in Fig. 4. These three pressures correspond to conditions below, at, and above [see Figs. 4(a), 4(b), and 4(c), respectively] the optimal condition at which $T_{c}$ is maximized. The main result that we observe is the appearance of an electron band crossing the Fermi level for the regular tetrahedron that induces the appearance of an electron pocket around the $Z$-point on the Brillouin zone. A careful analysis of the orbitals that give rise to this band shows that it is essentially a three-dimensional band that has an important contribution of the Sm orbitals.

The studied sample has a F content of $\sim 0.19$, i.e., a doping larger than the optimal one, ${ }^{27}$ and the sample is thus on the overdoped region. In this case, pressure would induce a monotonous reduction of $T_{c}$. At the optimum pressure, the $\mathrm{FeAs}_{4}$ tetrahedron is not distorted and the Sm band dips below the Fermi level, pumping electrons and reducing the doping towards the optimal level. In this way the maximum in $T_{c}$ can be simply explained in this case by charge transfer towards the optimal doping. A representative of the evolution of charges in the FeAs layer is shown in Fig. 4(d) (red filled circles), where it is clear that there is a reduction of the "number of electrons" at the optimum pressure in the FeAs block for the regular tetrahedron due to the lowering of the Sm band. The "electron concentration" was also calculated for a structure with the same lattice parameters but an irregular tetrahedron (with similar angles to the low-pressure case) showing that in such a case the evolution is monotonous [blue diamond in Fig. 4(d)]. Superconducting $T_{c}$ changes under pressure due to charge transfer under pressure have also been observed in 122 materials. $^{28}$

As our sample is in the overdoped region, there is no distortion to be expected below $T_{c}$, as reported for underdoped 122 materials. ${ }^{29}$ Thus, our calculation using the room temperature atomic positions at each pressure should be valid down to the lowest temperatures.

The decreasing measured pressure dependence of $T_{c}$ can be attributed to the combined effect of monotonous charge transfer and electronic susceptibility damping. The contribution of the Sm band adds just a small contribution that compensates for this effect only around the ideal tetrahedron configuration. This small contribution on a monotonous dependence explains the maximum.

\section{CONCLUSIONS}

In conclusion, correlated structural, transport, and electronic band properties of $\mathrm{SmFeAsO}_{0.81} \mathrm{~F}_{0.19}$ have been performed. No phase transition was observed up to $20 \mathrm{GPa}$ and the measured compressibility is similar to other compounds of the same family. This is, to our knowledge, the first pressure 
experiment done on the same sample where we show the importance of the structural parameters As height and AsFeAs angle on $T_{c}$. A maximum $T_{c}$ of $55.2 \mathrm{~K}$ is attained at the same pressure where the As atom height is $1.39 \AA$ and the AsFeAs angle reaches its nondistorted value of $109.47^{\circ}$.

It is furthermore shown that this $T_{c}$ maximum is due to an electron transfer to the $\mathrm{SmO}_{1-x} \mathrm{~F}_{x}$ plane changing the electron concentration in the FeAs planes from overdoped to optimally doped. As this picture is different from previously proposed scenarios, it suggests that the origin of the tetrahedron- $T_{c}$ relation can either have multiple origins or that the basic idea for the problem is still to be found. We hope that this work will stimulate theoretical groups to search for a general explanation, probably relying on symmetry rather than details of band calculations, which we suggest may be different for each compound.

\section{ACKNOWLEDGMENTS}

The authors express their thanks to J. Jacobs for the preparation of the diamond anvil cells and W. A. Crichton for the gas loading. This work was partially supported by the project SupraTetraFer ANR-09-BLAN-0211 of the Agence Nationale de la Recherche of France. R.W. is a member of CONICET-Argentina and gratefully acknowledges partial support from CONICET (Grant No. PIP 112-200801-00047) and ANPCyT (Grant No. PICT 837/07). *gaston.garbarino@esrf.fr

${ }^{1}$ Y. Kamihara, T. Watanabe, M. Hirano, and H. Hosono, J. Am. Chem. Soc. 130, 3296 (2008).

${ }^{2}$ H. Takahashi et al., Nature (London) 453, 376 (2008).

${ }^{3}$ X. H. Chen et al., Nature (London) 453, 761 (2008).

${ }^{4}$ M. Rotter, M. Tegel, and D. Johrendt, Phys. Rev. Lett. 101, 107006 (2008).

${ }^{5}$ A. Nazarenko and E. Dagotto, Phys. Rev. B 53, R2987 (1996).

${ }^{6}$ J. Ranninger and T. Domański, Phys. Rev. B 81, 014514 (2010).

${ }^{7}$ K. Kuroki, H. Usui, S. Onari, R. Arita, and H. Aoki, Phys. Rev. B 79, 224511 (2009).

${ }^{8}$ M. J. Calderón, B. Valenzuela, and E. Bascones, New J. Phys. 11, 013051 (2009).

${ }^{9}$ M. Núñez-Regueiro and C. Acha, in Studies of High Temperature Superconductors, edited by N. V. Narlikar (Nova Science, New York, 1997), Vol. 24, p. 203.

${ }^{10}$ B. Dabrowski, Z. Wang, K. Rogacki, J. D. Jorgensen, R. L. Hitterman, J. L. Wagner, B. A. Hunter, P. G. Radaelli, and D. G. Hinks, Phys. Rev. Lett. 76, 1348 (1996).

${ }^{11}$ O. Chmaissem et al., Nature 397, 45 (1999).

${ }^{12}$ C.-H. Lee et al., J. Phys. Soc. Jpn. 77, 083704 (2008).

${ }^{13}$ A. Kreyssig et al., Phys. Rev. B 78, 184517 (2008).

${ }^{14}$ S. A. J. Kimber et al., Nat. Mater. 8, 471 (2008)

${ }^{15}$ J. Zhao, Q. Huang, C. de la Cruz, S. Li, J. W. Lynn, Y. Chen, M. A. Green, G. F. Chen, G. Li, Z. Li, J. L. Luo, N. L. Wang, and P. Dai, Nat. Mater. 7, 953 (2008).

${ }^{16} \mathrm{P}$. Quebe, L. J. Terbüchte, and W. Jeitschko, J. Alloys Compd. 302, 70 (2000).
${ }^{17}$ R. H. Liu, G. Wu, T. Wu, D. F. Fang, H. Chen, S. Y. Li, K. Liu, Y. L. Xie, X. F. Wang, R. L. Yang, L. Ding, C. He, D. L. Feng, and X. H. Chen, Phys. Rev. Lett. 101, 087001 (2008).

${ }^{18}$ A. P. Hammersley et al., High Pressure Res. 14, 235 (1996).

${ }^{19}$ A. C. Larson and R. B. Von Freele, Los Alamos National Laboratory Report LUAR (1994), pp 86-748; B. H. Toby, J. Appl. Crystallogr. 34, 210 (2001).

${ }^{20}$ S. Sanfilippo, H. Elsinger, M. Núñez-Regueiro, O. Laborde, S. LeFloch, M. Affronte, G. L. Olcese, and A. Palenzona, Phys. Rev. B 61, R3800 (2000).

${ }^{21}$ G. Garbarino, P. Toulemonde, M. Alvarez-Murga, A. Sow, M. Mezouar, and M. Núñez-Regueiro, Phys. Rev. B 78, 100507 (2008).

${ }^{22}$ F. Birch, J. Geophys. Res. 83, 1257 (1978).

${ }^{23}$ A. Martinelli, M. Ferretti, A. Palenzona, and M. Merlini, Physica C 469, 782 (2009).

${ }^{24}$ R. Jeanloz and R. M. Hazen, Am. Mineral. 76, 1765 (1991).

${ }^{25}$ P. Blaha, K. Schwarz, G. K. H. Madsen, D. Kvasnicka, and J. Luitz, in wien $2 k$, An Augmented Plane Wave and Local Orbitals Program for Calculating Crystal Properties, edited by K. Schwarz (Vienna University of Technology, Vienna, 2001).

${ }^{26}$ J. P. Perdew, K. Burke, and M. Ernzerhof, Phys. Rev. Lett. 77, 3865 (1996).

${ }^{27}$ Y. Kamihara et al., New J. Phys. 12, 033005 (2010).

${ }^{28}$ M. L. Gooch, B. Lv, B. Lorenz, A. M. Guloy, and C.-W. Chu, Phys. Rev. B 78, 180508 (2008).

${ }^{29}$ S. Nandi, M. G. Kim, A. Kreyssig, R. M. Fernandes, D. K. Pratt, A. Thaler, N. Ni, S. L. Bud'ko, P. C. Canfield, J. Schmalian, R. J. McQueeney, and A. I. Goldman, Phys. Rev. Lett. 104, 057006 (2010). 